\title{
Effects of tip separation and orientation on negative differential resistance in boron-doped carbon-nanotube-based molecular junctions
}

\author{
ZHAO Peng $^{1 *}$, LIU DeSheng $^{2} \&$ LIANG Wei ${ }^{1}$ \\ ${ }^{1}$ School of Physics and Technology, University of Jinan, Jinan 250022, China; \\ ${ }^{2}$ School of Physics, State Key Laboratory of Crystal Materials, Shandong University, Jinan 250100, China
}

Received October 3, 2011; accepted November 28, 2011

\begin{abstract}
We investigate using the Landauer formalism, which combines both the non-equilibrium Green's function and density functional theory, the effects of separation and orientation between two electrodes of boron-doped capped-carbon-nanotube-based molecular junctions on negative differential resistance. The results show that this negative differential resistance behavior is strongly dependent on the separation and orientation between the two electrodes. A gap width of $0.35 \mathrm{~nm}$ and maximal symmetry achieves the best negative differential resistance behavior.
\end{abstract}

carbon nanotube, negative differential resistance, non-equilibrium Green function, density functional theory

Citation: Zhao P, Liu D S, Liang W. Effects of tip separation and orientation on negative differential resistance in boron-doped carbon-nanotube-based molecular junctions. Chin Sci Bull, 2012, 57: 966-969, doi: 10.1007/s11434-012-4972-y

In recent years, progress in techniques for characterizing and manipulating individual molecules has made it possible to develop single molecular devices [1]. A number of interesting physical properties, including negative differential resistance (NDR) [2-10], single-electron characteristics [11], highly nonlinear current-voltage $(I-V)$ relationships [12], rectification [13], and so on, have been found in various kinds of single molecular junctions. The most prominent among these is the NDR effect, which features a rise and fall in current as the applied bias voltage is steadily increased. NDR is a very useful property due to its utility in molecular devices such as molecular switches, logic cells and memory storage. NDR behavior has been found in many systems, such as oligo(phenylene ethynylene) (OPE) molecular junction with nitro and amino groups on the central phenyl ring [2], porphyrin junction with side groups [3], anthracene junction [4], metallic and semiconducting clusters [5], nitrogen-terminated single-wall carbon nanotube (SWCNT) junction [6], and $\mathrm{C}_{121}$ [7] and $\mathrm{C}_{131}$ [8] molecular

*Corresponding author (email: ss_zhaop@ujn.edu.cn) junctions.

In a previous study, we performed a theoretically study of the electron transport properties of boron-doped cappedSWCNT junctions [14]. Our results showed that their electron transport properties are strongly dependent on the dopant site, namely, $a, b, c, d$, and $e$ sites (see Figure 1). Moreover, NDR behavior can be observed if boron is present in the tip region, i.e. $a, b$, and $c$ sites; in particular, the $c$ doping site gives rise to the highest peak-to-valley ratio (PVR). Because no bridging molecule is used, the structure of this NDR device is simpler compared with ordinary molecular NDR devices.

It is well known that the distance between electrodes can strongly affect the electron transport properties of a molecular junction $[15,16]$. Recent advances in nanofabrication techniques permit a precise control of the gap size in between the SWCNT electrodes [17]. One fundamental though intriguing question arises: how does the distance between two SWCNT electrodes affect the NDR behavior in the boron-doped capped-SWCNT junction? Moreover, it is also interesting to explore the effect of orientation between two 


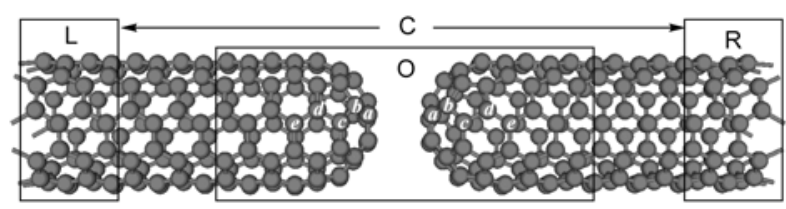

Figure 1 Schematic illustration of the capped-SWCNT junction showing dopant sites and division into regions for calculation purposes. In our studies boron atoms occupy the $c$-site.

SWCNT electrodes on the NDR behaviour [15]. With this aim, we performed a theoretical simulation using the Landauer formalism that combines both the non-equilibrium Green's function (NEGF) and density functional theory (DFT) to study these effects.

Figure 1 shows a schematic of a boron-doped cappedSWCNT $(5,5)$ junction. Both capped-SWCNT(5,5) electrodes are closed with fullerene hemispheres with opposing boron atoms at the $c$ sites. In theoretical simulations, the molecular junction is divided into three regions: the left semi-infinite electrode (L), the central scattering region (C), and the right semi-infinite electrode $(\mathrm{R})$. The tip gap width, i.e. the distance $w$ between the two tips, is set at $0.20,0.25$, 0.35 and $0.40 \mathrm{~nm}$, respectively. As stated in our previous work [14], the structures comprising the $\mathrm{O}$ region have been optimized using the SIESTA package [18], and the electron transport calculations have been performed using the ATK package $[19,20]$, which combines DFT and NEGF.

Figure 2 shows the calculated $I$ - $V$ curves for each junction for pre-set tip gap width. The bias voltages are in the range $0-1.5 \mathrm{~V}$, which is reasonable for experimental meas-
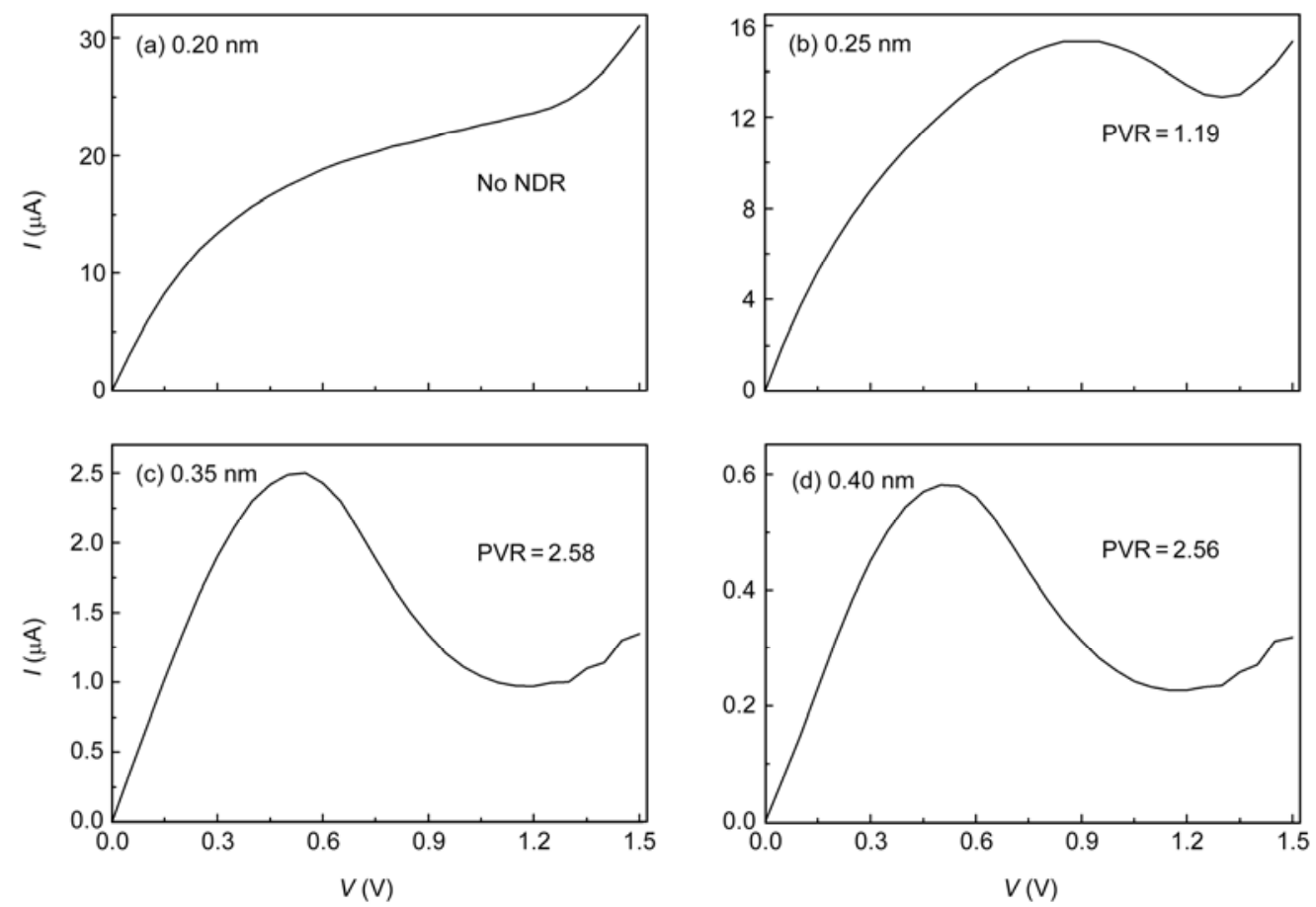

urements [21]. From the $I-V$ curves, it can be seen that the current decreases dramatically with increasing tip gap over the whole bias voltage range. In addition, we can see that tip gap width strongly affects the NDR behavior: the PVR weakens when this width decreases from 0.35 to $0.25 \mathrm{~nm}$, and it vanishes completely at $0.20 \mathrm{~nm}$; the PVR drops slightly as the width increases from 0.35 to $0.40 \mathrm{~nm}$. As a result, a width of $0.35 \mathrm{~nm}$ gives a relatively good NDR behavior with regard to peak height and PVR value.

To understand the effect of width on NDR behavior, we calculate the transmission spectrum of our molecular junctions setting $w=0.20,0.35 \mathrm{~nm}$ with bias voltage $0,0.20$, $0.55,0.90$ and $1.20 \mathrm{~V}$, as shown in Figure 3. The current is calculated using the Landauer-Büttiker formula [22]:

$$
I=(2 e / h) \int_{\mu_{\mathrm{L}}}^{\mu_{\mathrm{R}}}\left(f_{\mathrm{L}}-f_{\mathrm{R}}\right) T(E, V) \mathrm{d} E,
$$

where $\mu_{\mathrm{L}, \mathrm{R}}=E_{\mathrm{F}} \mp \mathrm{eV} / 2, E_{\mathrm{F}}$ is the Fermi level, $f$ the Fermi distribution function, and $T(E, V)$ the bias-dependent- transmission function. Consequently, only electrons with energies within the energy region, $\left[\mu_{\mathrm{L}}, \mu_{\mathrm{R}}\right]$, contribute to the total current; this region is called the bias or integral window. Setting $E_{\mathrm{F}}$ to zero, the bias window actually covers the region $[-V / 2, V / 2]$. Thus, the current is determined by the integral of $T(E, V)$ over the bias window. For the junction with width $w=0.20 \mathrm{~nm}$ (Figure 3(a)), there is always a strong and broad peak in the bias window, which remains essentially unchanged as bias voltage increases. As a result, current will continually increase and NDR behavior will not be present. In contrast, for the junction with width $w=0.35 \mathrm{~nm}$

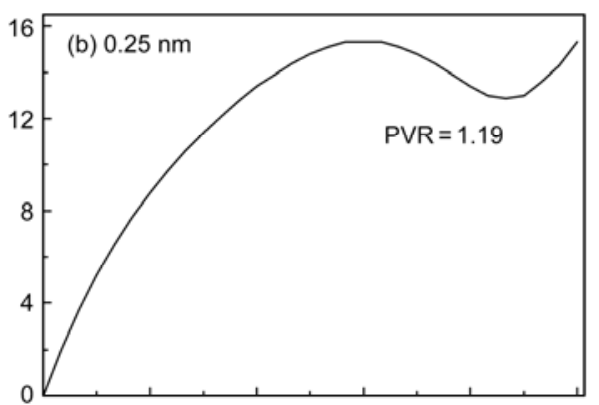

Figure $2 I-V$ curves of junctions with preset tip gap widths. 

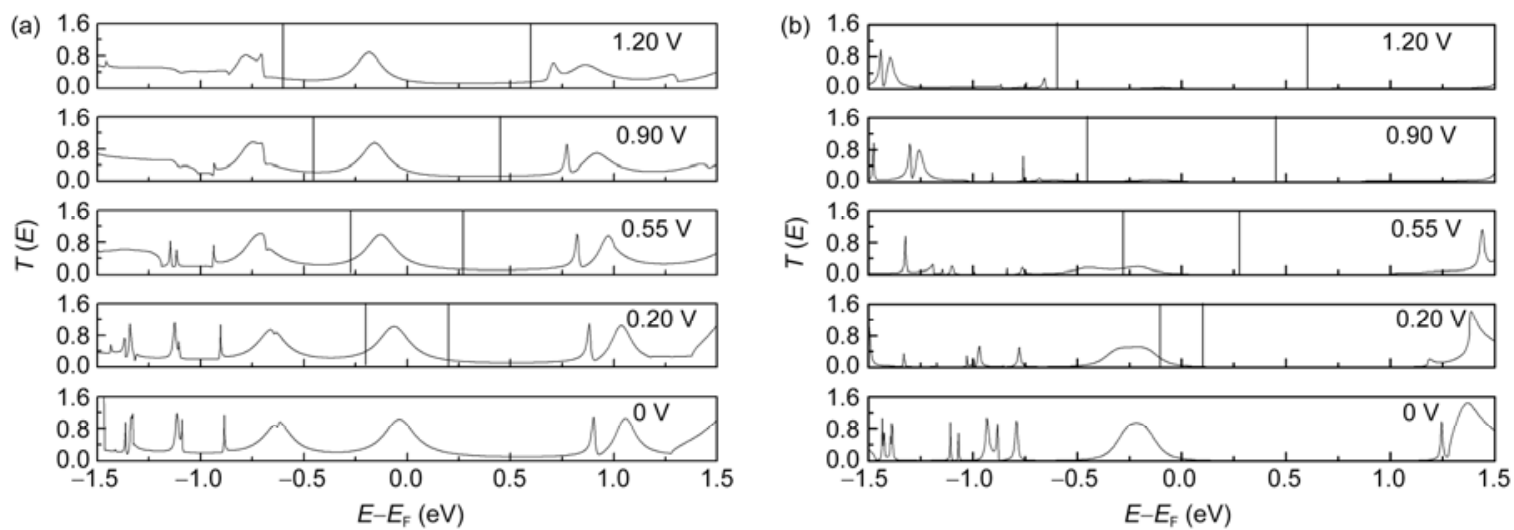

Figure 3 Calculated transmission spectra of the molecular junctions with widths (a) $w=0.20 \mathrm{~nm}$, (b) $w=0.35 \mathrm{~nm}$ under bias voltages $0,0.20,0.55,0.90$ and $1.20 \mathrm{~V}$. The region between the two vertical solid lines indicates the bias window.

(Figure 3(b)), there is also a strong and broad peak just below $E_{\mathrm{F}}$ under small bias voltages, resulting in an initial increase in current. However, the applied bias voltage simultaneously reduces the peak height. The overall reduction in peak height suppresses any gain resulting from a wider bias window, leading to a net drop in current as the bias voltage exceeds $0.55 \mathrm{~V}$; this suppression constitutes the NDR behavior.

To understand the different transmission characteristics with tip gap width, we project the density of states (PDOS) onto the $\mathrm{C}$ regions of the left (solid line) and right (dashed line) tubes of the molecular junctions for widths $w=0.20$, $0.35 \mathrm{~nm}$ under bias voltages 0 and $1.20 \mathrm{~V}$ [7], as shown in Figure 4. From the PDOS spectra, we can see that these two sets of PDOS peaks are degenerate in energy in the zerobias case, and there are obvious PDOS peaks just below $E_{\mathrm{F}}$ that give rise to strong and broad transmission peaks in Figure 3 near $E_{\mathrm{F}}$. If a $1.20 \mathrm{~V}$ bias voltage is applied, then for the junction with small gap width (Figure 4(a)), these two sets of PDOS peaks are still nearly degenerate in energy near $E_{\mathrm{F}}$. As a result, the peak in the bias window does not change substantially as bias voltage increases (Figure 3(a)). In contrast, for the junction with larger gap width (Figure 4(b)), these two sets of PDOS peaks are non-degenerate in energy near $E_{\mathrm{F}}$ if a $1.2 \mathrm{~V}$ bias voltage is applied. Consequently, there is no obvious transmission peak near $E_{\mathrm{F}}($ Figure 3(b)).

Finally, we explore the effect of orientation between the two SWCNT electrodes on the NDR behavior by rotating the right tube around its symmetry axis while maintaining a fixed tip gap width $(0.35 \mathrm{~nm})$ [15]. Clearly the rotation reduces the symmetry of the molecular junction. We present in Figure 5 the corresponding $I-V$ curves and PVR values for different rotation angles; these curves show a strong angular dependence in NDR behavior. The PVR value drops from 2.58 to 1.09 over an angular range $0^{\circ}-72^{\circ}$, and vanishes as the rotation angle exceeds $72^{\circ}$. Thus, the maximal symmetry, i.e. opposing boron atoms, gives rise to the best NDR behavior for our junctions.
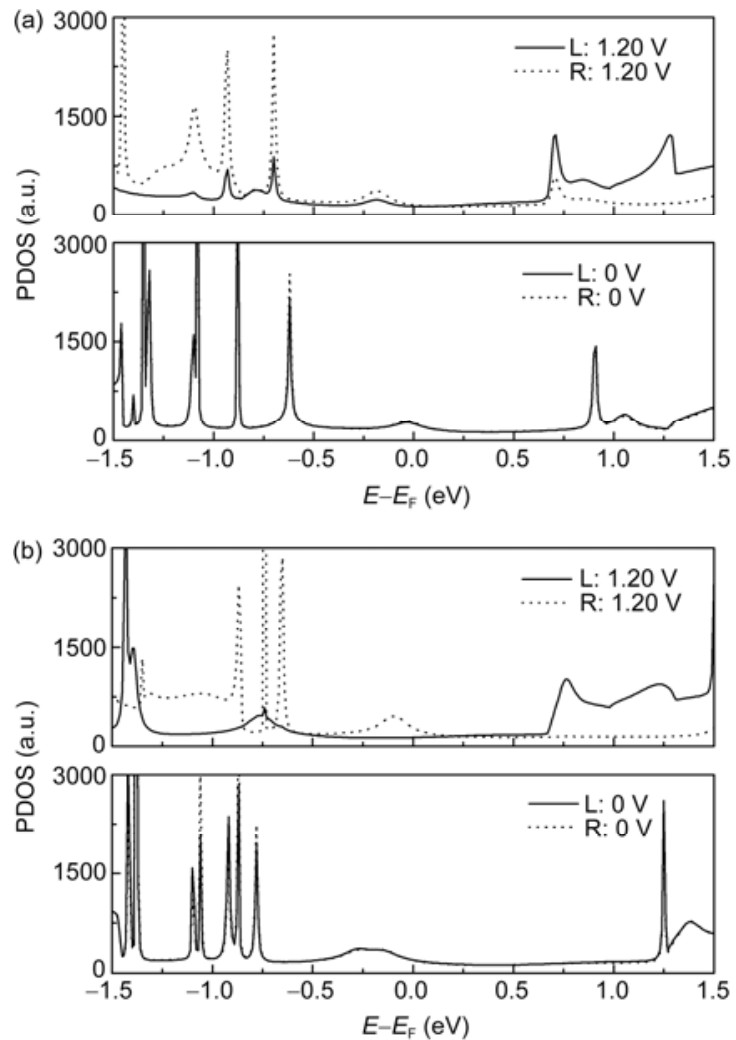

Figure 4 The PDOS of the left (solid line) and right (dashed line) tube of the $\mathrm{C}$ region in the molecular junctions for (a) $w=0.20 \mathrm{~nm}$, (b) $w=0.35 \mathrm{~nm}$ under bias voltages 0 and $1.20 \mathrm{~V}$. The characters $\mathrm{L}$ and $\mathrm{R}$ identify the left and right tube.

In conclusion, we have investigated the effects of tip gap width and orientation between the two electrodes on the NDR behavior of boron-doped capped-SWCNT junctions. The results showed that the NDR behavior is strongly dependent on the width and orientation between the two SWCNT electrodes. A width of $0.35 \mathrm{~nm}$ and maximal symmetry gives rise to the best NDR behavior. We believe that our results will be helpful for the application of the SWCNTs in the field of NDR devices. 


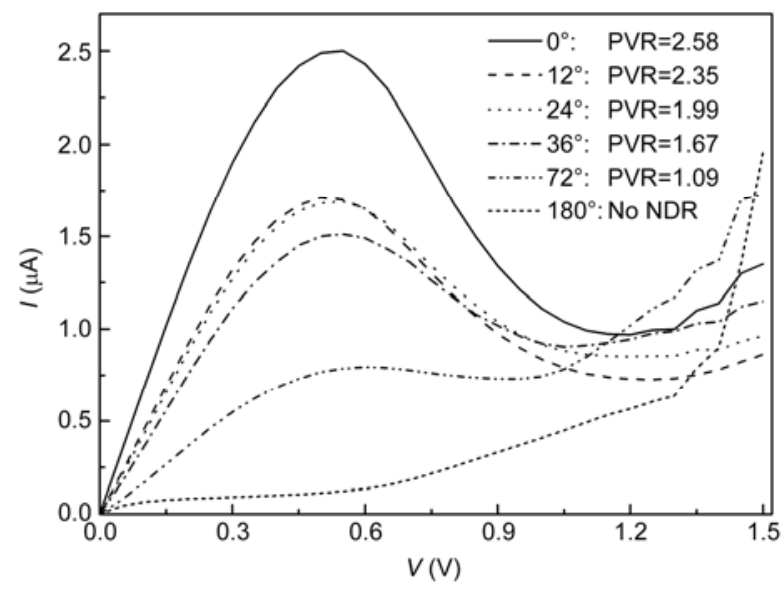

Figure $5 I-V$ curves for six different rotation angles of the right electrode around its symmetry axis. The tube-tube separation $w$ is fixed at $0.35 \mathrm{~nm}$.

This work was supported by the National Natural Science Foundation of China $(11104115,11074146)$, the Natural Science Foundation of Shandong Province of China (ZR2009AL004), the Doctoral Foundation of University of Jinan (XBS1004) and the Key Laboratory of Semiconductor Materials Science Program of Institute of Semiconductors of Beijing (KLSMS-0908).

1 Andres R P, Bein T, Dorogi M, et al. Science, 1996, 272: 1323-1325

2 Chen J, Reed M A, Rawlett A M, et al. Science, 1999, 286:
$1550-1552$

3 Long M Q, Chen K Q, Wang L L, et al. Appl Phys Lett, 2008, 92: 243303

4 Zhao P, Fang C F, Xia C J, et al. Appl Phys Lett, 2008, 93: 013113

5 Seminario J M, Araujo R A, Yan L M, et al. J Phys Chem B, 2004, 108: 6915-6918

6 Zhao P, Wang P J, Zhang Z, et al. Phys Lett A, 2010, 374: 11671171

7 Zhao P, Liu D S, Zhang Y, et al. Phys Lett A, 2011, 375: 2639-2643

8 Zhao P, Liu D S. Solid State Commun, 2011, 151: 1424-1427

9 Ma J S, Li D M, Zhai Y X, et al. Sci China Phys Mech Astron, 2011, 54: $1433-1437$

10 Yang Y T, Song J X, Liu H X, et al. Chin Sci Bull, 2008, 53: 3770-3772

11 Kelly T R, Silva H D, Silva R A. Nature, 1999, 401: 150-152

12 Crljen Ž, Grigoriev A, Wendin G, et al. Phys Rev B, 2005, 71: 165316

13 Ng M K, Lee D C, Yu L P. J Am Chem Soc, 2002, 124: 1186211863

14 Zhao P, Liu D S. Chin Sci bull, 2010, 55: 4104-4107

15 Zhao P, Wang P J, Zhang Z, et al. Chin Sci Bull, 2010, 55: 12271230

16 Ji G M, Zhai Y X, Fang C F, et al. Phys Lett A, 2011, 375: 16021607

17 Wei D C, Liu Y Q, Cao L C, et al. Nano Lett, 2008, 8: 1625-1630

18 Soler J M, Artacho E, Gale J D, et al. J Phys: Condens Matter, 2002, 14: $2745-2779$

19 Taylor J, Guo H, Wang J. Phys Rev B, 2001, 63: 245407

20 Brandbyge M, Mozos J L, Ordejón P, et al. Phys Rev B, 2002, 65: 165401

21 Reed M A, Chen J. Chem Phys, 2002, 281: 127-145

22 Büttiker M, Imry Y, Landaur R, et al. Phys Rev B, 1985, 31: 62076215

Open Access This article is distributed under the terms of the Creative Commons Attribution License which permits any use, distribution, and reproduction in any medium, provided the original author(s) and source are credited. 